\title{
Diffusion of Bovine Serum Albumin in a Neutral Polymer Solution
}

\author{
GEORGE D. J. PHILLIES, Department of Chemistry, The University \\ of Michigan, Ann Arbor, Michigan 48109
}

\section{Synopsis}

The diffusion coefficient $D$ of bovine serum albumin through various solutions ( $\mathrm{pH}$ $7.0,0.5 \mathrm{M} \mathrm{NaCl})$ of polyethylene oxide $\left(M_{W} \sim 1 \times 10^{5}, 3 \times 10^{5}\right)$ was studied with quasielastic light scattering. In solutions of the $1 \times 10^{5}$ polymer solution at polymer concentrations above $0.5 \mathrm{~g} / \mathrm{L}, D$ is considerably greater than would have been expected from the viscosity of water:polymer mixtures, the deviations being larger at low protein concentration than at high protein concentration. With either polymer, $D$ falls with increasing protein concentration.

\section{INTRODUCTION}

Hydrodynamic studies of biopolymers have in large part been concerned with the behavior of single species in dilute solution, especially as an approach to the determination of molecular size and shape. Some macromolecules (e.g., hemoglobin) naturally occur at high concentration; a significant number of studies on the hydrodynamic properties of concentrated solutions of a single biopolymer (e.g., hemoglobin, ${ }^{1-6}$ bovine serum albumin ${ }^{6-8}$ ) have been reported. There are also a number of cases in which one is interested in the motion of a given species through a solvent containing substantial amounts of a mixture of other biopolymers. Such a circumstance arises in the laboratory in chromatography and gel electrophoresis, in which the diffusion of macromolecules through a polymeric matrix determines the resolution of the procedure; such circumstances are also found within the living cell, in which macromolecuies exhibit their activities and movements while bathed in a highly concentrated mixture of biopolymers.

Few studies have been made of the diffusion of biopolymers through a mixed macromolecule solution. One notes the work of Langevin and Rondele $z^{9}$ (on systems similar to ours), Brown and Stilbs ${ }^{10}$ (on selfdiffusion in polyethylene oxide), Turner and Hallet ${ }^{11}$ (on polystyrene spheres in water:dextran), Laurent et al. ${ }^{12-14}$ (on proteins and other probe particles in water:dextran), and this author and coworkers [on spherical probe particles in solutions of poly-(acrylic acid) ${ }^{15-17}$ and polyethylene oxide ${ }^{18}$. Much of this work used large $(200 \AA-2 \mu \mathrm{m})$ probe particles, though Laurent et al. and Langevin and Rondelez did report the use of protein molecules as probes. Laurent and Persson ${ }^{12}$ reported solution viscosities, as well as sedimentation coefficients $s$, finding that 
$s_{0} / s$ increases with concentration more slowly than does $\eta / \eta_{0}$; i.e., probe particles in water:dextran sediment more quickly than would be expected from the macroscopic shear viscosity. Here, the subscript " 0 " denotes parameters measured in dextran-free solutions.

Data are here reported on the diffusion of coefficient $D$ of bovine serum albumin (at concentration $C_{\mathrm{BSA}}$ ) moving through solutions of polyethylene oxide (concentration $C_{\mathrm{PEO}}$ ) in $0.5 \mathrm{M} \mathrm{NaCl}$, pH 7.0. Comparison is made with the measured viscosity of water:polyethylene oxide; the results are analyzed in terms of scaling-type theories of polymer solutions.

\section{EXPERIMENTAL METHODS}

The polymers studied were polyethylene oxides (Polysciences, Inc.) of molecular weights $1 \times 10^{5}$ and $3 \times 10^{5}$. These were used to prepare stock solutions (in $0.5 \mathrm{M} \mathrm{NaCl}$ ) of polymer concentrations 5.0 and 2.5 $\mathrm{g} / \mathrm{L}$, respectively, the latter concentration being fixed by the solubility limit of the $3 \times 10^{5}$ amu polymer. The $1 \times 10^{5}$ amu polyethylene oxide gave a water-white transparent stock solution, but even at half the concentration, the $3 \times 10^{5}$ amu polymer yielded a faintly hazy stock solution. The light-scattering spectrum of the $3 \times 10^{5}$ polymer, while detectable, was substantially weaker than the scattering spectrum of the protein solutions. To be conservative, the scattering spectra of serum albumin in the $3 \times 10^{5} \mathrm{amu}$ polymer for $C_{\mathrm{BSA}}<33 \mathrm{~g} / \mathrm{L}$ is not reported. Viscosities of water:polyethylene oxide solutions had previously been determined for an independent study ${ }^{18}$ of probe diffusion by polystyrene spheres through water:polyethylene oxide.

Bovine serum albumin (Sigma, essentially fatty-acid free) was added to mixtures of $0.5 \mathrm{M} \mathrm{NaCl}$ and the polymer solutions to final protein concentrations of $10,33,50$, and $67 \mathrm{~g} / \mathrm{L}$. The protein was allowed to dissolve and was then passed through $0.2-\mu \mathrm{m}$ Nucleopore filters into the cleaned scattering cells, which were $1-\mathrm{cm}$ glass fluorimeter cells, four sides polished. The cells were placed in temperature-controlled $\left(25 \pm 0.1^{\circ} \mathrm{C}\right)$ mounts and allowed to come to thermal equilibrium. The solution $\mathrm{pH}$ was 7.0 .

In the spectrometer, samples were illuminated with a $20-\mathrm{mW} \mathrm{He}-$ $\mathrm{N}$ laser; the scattered light was allowed to fall on the surface of an RCA 7265 photomultiplier tube. The photopulses were analyzed with 64- and 128-channel Langley-Ford digital correlators. Correlation functions $S(k, t)$ were analyzed against a cumulant expansion,

$$
1 / 2 \ln [S(k, t)-B]=\sum_{i=0}^{n} K_{i}(-t)^{i} / i !
$$

where $B$ is the baseline obtained from the correlator delay channels, $K_{i}$ is the $i$ th cumulant, and $n$ is the order of the fit, using standard statistical tests to select the best value (usually $n=2$ ) for $n$. 


\section{RESULTS}

Figure 1 gives $D$ as a function of $C_{\mathrm{PEO}}$ for the $1 \times 10^{5}$ amu polymer. At each protein concentration, $D$ is observed to fall as the polymer concentration is increased; the dependence of $D$ on $C_{\mathrm{PEO}}$ varies with $C_{\mathrm{BSA}}$. In the $1 \times 10^{5}$ polymer, with increasing $C_{\mathrm{PEO}}, D$ for $10 \mathrm{~g} / \mathrm{L}$ of BSA falls from 6.7 to $\sim 6.1 \times 10^{-7} \mathrm{~cm}^{2} / \mathrm{s}$ and then levels off. With 33 $\mathrm{g} / \mathrm{L}$ of BSA, $D$ falls by nearly $20 \%$ over the polymer concentration range $0-5 \mathrm{~g} / \mathrm{L}$; with $50 \mathrm{~g} / \mathrm{L}$ of BSA, $D$ falls by more than $25 \%$ over the same range of polymer concentrations.

Figure 2 shows the dependence of $D$ on the concentration of $3 \times$ $10^{5}$ amu polyethylene oxide. $D$ falls steeply with increasing $C_{\text {PEO }}$, so that for any $C_{\mathrm{BSA}}, D$ in $2.5 \mathrm{~g} / \mathrm{L}$ of the $3 \times 10^{5}$ amu polymer is less than $D$ in $5.0 \mathrm{~g} / \mathrm{L}$ of the $1 \times 10^{5}$ polymer. While $D$ does fall with increasing protein concentration, the dependence of $D$ on $C_{\mathrm{BSA}}$ is quite weak. In comparing this result with the strong dependence of $D$ on $C_{\mathrm{BSA}}$ in the $1 \times 10^{5}$ amu polymer solutions, it is important to recognize that $D$ 's dependence on $C_{\mathrm{BSA}}$ in the $1 \times 10^{5}$ system is most evident at large $(>2 \mathrm{~g} / \mathrm{L})$ polymer concentrations. Because of the solubility limit of the polymer in water, these values of $C_{\mathrm{PEO}}$ could not be reached with the $3 \times 10^{5}$ amu polymer.

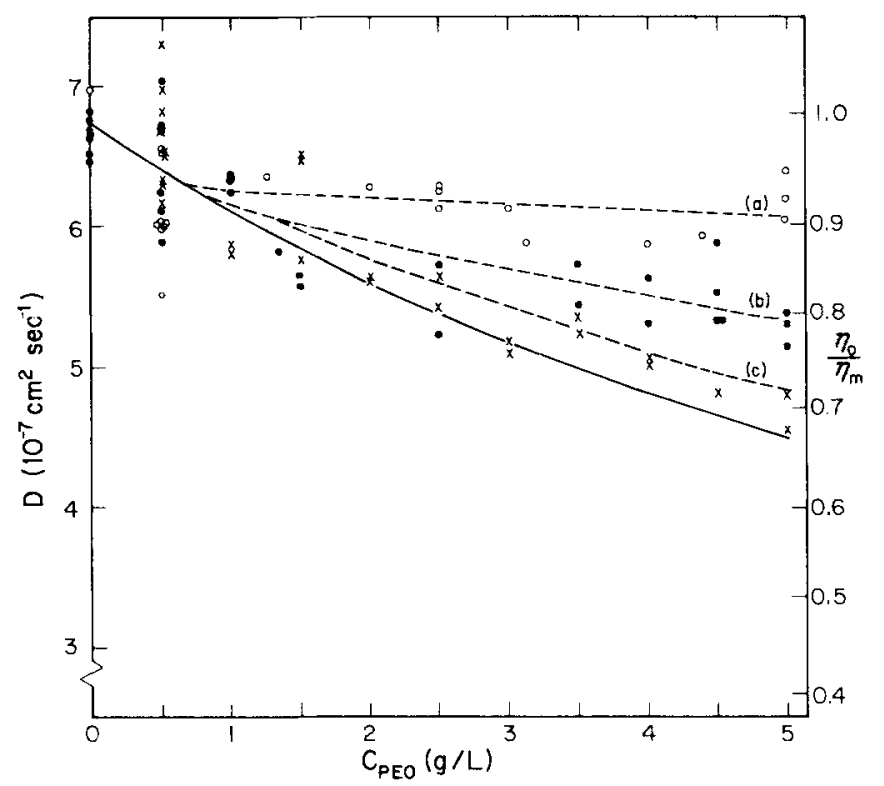

Fig. 1. Mutual diffusion coefficient (in units $10^{-7} \mathrm{~cm}^{2} \mathrm{~s}^{-1}$ ) of bovine albumin in water: $1 \times 10^{5} \mathrm{amu}$ polyethylene oxide: $0.5 \mathrm{M} \mathrm{NaCl}, \mathrm{pH} 7$, plotted against the concentration $(g / L)$ of polyethylene oxide. Measurements were made at protein concentrations of $10(\mathrm{O}), 33(-)$, and $50(\times) \mathrm{g} / \mathrm{L}$. The solid line denotes the solution fluidity; dashed lines mark fits of the (a) $10 \mathrm{~g} / \mathrm{L}$, (b) $33 \mathrm{~g} / \mathrm{L}$, and (c) $50 \mathrm{~g} / \mathrm{L}$ data to the form $D / D_{0}=$ $\exp \left(-a C^{v}\right) ; \nu$ and $\alpha$ are given in Table $\mathbf{I}$. 


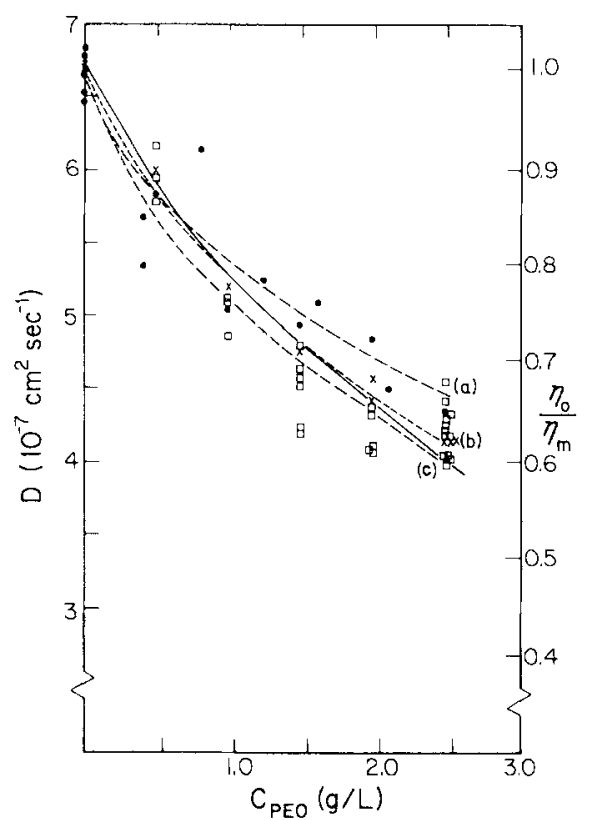

Fig. 2. Mutual diffusion coefficient (in units $10^{7} \mathrm{~cm}^{2} \mathrm{~s}^{-1}$ ) of bovine serum albumin in water: $3 \times 10^{5}$ amu polyethylene oxide: $0.5 \mathrm{M} \mathrm{NaCl}, \mathrm{pH} 7$, as a function of the polyethylene oxide concentration. Measurements were made at protein concentrations of $33(0), 50(x)$, and $67(\square), g / L$. The solid line marks the solution fluidity; dashed lines represent fits of the (a) $33 \mathrm{~g} / \mathrm{L}$, (b) $50 \mathrm{~g} / \mathrm{L}$, and (c) $67 \mathrm{~g} / \mathrm{L}$ data to the form $D / D_{0}=$ $\exp \left(-a C^{v}\right)$, where $\nu$ and $a$ may be found in Table I. Line (b) is indistinguishable from solution fluidity.

For reasons that are not entirely clear, the scatter in measurements of $D$ in polymer solutions is appreciably larger than might be expected from data in pure water. With the $1 \times 10^{5} \mathrm{amu}$ polymer, the reproducibility in $D$ is usually better than $\pm 0.15 \times 10^{-7} \mathrm{~cm}^{2} \mathrm{~s}^{-1}$. On a substantial number of repetitions, the scatter in $D$ for $C_{\mathrm{PEO}}$ near 0.5 $\mathrm{g} / \mathrm{L}$ is much worse than at other polymer concentrations. The scatter in values of $D$ is larger with the $3 \times 10^{5}$ amu polymer than with the $1 \times 10^{5}$ amu polymer.

Figures 1 and 2 also show (solid lines) the fluidities $\left(\eta^{-1}, \eta\right.$ being the macroscopic shear viscosity) of the polymer solutions, in units such that $\eta^{-1}=\eta_{0}^{-1}$ corresponds to $D=D_{0}$. The viscosity $\eta$ is linear in $C_{\mathrm{PEO}}$. At a fixed temperature, as used here, the Stokes-Einstein equation predicts $D \sim \eta^{-1}$. In the $1 \times 10^{5}$ amu polymer, $D$ is consistently larger (in some cases more than a third larger) than expected from the $C_{\mathrm{PEO}}$ dependence of $\eta$. In the $3 \times 10^{5}$ amu polymer, the behavior of $D$ for the more concentrated protein solutions is not greatly different from the behavior of $\eta^{-1}$ 


\section{DISCUSSION}

In small-molecule solvents such as water, the translational diffusion coefficient of a spherical macroparticle is given accurately by the Stokes-Einstein equation,

$$
D=k_{B} T / 6 \pi \eta R
$$

where $k_{B}$ is Boltzmann's constant, $\eta$ is the viscosity, and $R$ is the sphere's hydrodynamic radius. This equation was historically obtained for a continuum solvent; it need not necessarily be valid in a polymer solution, in which entanglements or non-Newtonian viscosity phenomena might be important. The results for serum albumin in the $1 \times$ $10^{5} \mathrm{amu}$ polymer solutions tend to sustain this concern, in that diffusion through these solutions is appreciably more rapid than predicted by Eq. (1). Polymer adsorption by the probes cannot explain this deviation, since adsorption would reduce, rather than increase $D$. On the other hand, serum albumin in $3 \times 10^{5}$ polyethylene oxide diffuses at roughly the rate to be expected from the macroscopic shear viscosity. Because the accessible ranges of $C_{\mathrm{PEO}}$ are not the same for the $1 \times$ $10^{5}$ and $3 \times 10^{5}$ polymers, the significance of difference between trends in $D$ in these two solutions should not be overstated.

Failures in the Stokes-Einstein equation are sometimes parameterized in terms of a microviscosity $\eta_{m}$,

$$
\eta_{m}=K_{B} T / 6 \pi R D
$$

which may be calculated from the known experimental parameters $T$, $R$, and $D$. By direct substitution,

$$
\eta_{0} / \eta_{m}=D / D_{0}
$$

The right-hand sides of Figs. 1 and 2 give $\eta_{0} / \eta_{m}$.

The only published experimental results that are directly comparable with our own are those of Langevin and Rondelez, ${ }^{9}$ who measured the sedimentation coefficient $s$ of serum albumin and other materials through solutions of polyethylene oxides of molecular weights $1.4 \times$ $10^{5}, 3 \times 10^{5}$ and $3.37 \times 10^{5} . \mathrm{s} / \mathrm{s}_{0}$ was found to depend on $C_{\mathrm{BSA}}$. On observing serum albumin at $C_{\mathrm{BSA}}=0.09,1$ and $5 \mathrm{~g} / \mathrm{L}$, and extrapolating to $C_{\mathrm{BSA}}$ to 0 , they found that $s / s_{0}=\exp \left(-a C_{\mathrm{PEO}}^{\nu}\right)$ for $v=0.52$ $\pm 0.20 . s$ was found to be independent of the polymer molecular weight $M$. As discussed below, our data also fit a power-law form with a similar value of $\nu$; however, at fixed $C_{\mathrm{PEO}}$, we find that $D / D_{0}$ depends substantially on $M$, regardless of whether one compares measurements at the same $C_{\mathrm{BSA}}$ or extrapolates $C_{\mathrm{BSA}}$ to 0 . Langevin and Rondelez do 
not report the viscosity of their solutions, so their sedimentation values cannot be used to compute the apparent hydrodynamic radii $k_{B} T /$ $6 \pi \eta D$ of their probes.

Theoretical studies of probe diffusion in polymer solutions are few in number. The classic formula of Ogston et al., ${ }^{19}$

$$
D / D_{0}-\exp \left[-a R C^{1 / 2}\right]
$$

$C$ being the polymer concentration, applies to diffusion through a random lattice of rigid rods, so its applicability to our systems is questionable. Langevin and Rondelez $z^{9}$ cite scaling arguments for the form

$$
D / D_{0}=\psi(R / \xi)
$$

where $\psi$ is an (unspecified) univeral function of the dimensionless parameter $R / \xi$. $\xi$ is the polymer scaling length, which is usually interpreted as the distance between entanglement points in the polymer. Recently, Cukier ${ }^{20}$ proposed the explicit form

$$
D / D_{0}=\exp (-\kappa R)
$$

where $\kappa$ is the hydrodynamic screening length in the solution. $\kappa$ depends on the polymer concentration $C$; for a coarse-grained analysis, an ideal polymer chain, and a swollen chain, the Debye-Bueche-Brinkman, ${ }^{21,22}$ Edwards-Freed, ${ }^{23,24}$ and deGennes ${ }^{25}$ approaches give $\kappa \sim C^{1 / 2}$, $C$, and $C^{3 / 4}$, respectively. Cukier's theory ${ }^{20}$ is applicable in the range $\kappa R \lesssim 1$, for polymer solutions more concentrated than a critical overlap concentration $C^{*}$. The systems studied here satisfy these requirements. Namely, from the work of Langevin and Rondelez, ${ }^{9} C^{*} \sim 0.3$ $0.5 \mathrm{~g} / \mathrm{L}$ for polyethylene oxides in our molecular-weight ranges.

Furthermore, $\kappa$ is essentially the same as the deGennes screening length $\xi$. From Langevin and Rondelez, ${ }^{9}$ one has $\xi \sim C^{-0.67}$ (where the possible error in the exponent is $\sim 0.1$ ); at a concentration of $10 \mathrm{~g} / \mathrm{L}$, $\xi$ is $\sim 45 \AA$. For serum albumin with an apparent hydrodynamic radius (as calculated from $D$ ) of $37 \AA$, this paper reports on systems with $\kappa R$ $\sim 0.18-0.52$. Most recently, Altenberger and Tirrell ${ }^{26}$ predicted that the lead term in the concentration dependence of $D$ in a gel is proportional to $C^{1 / 2}$.

We fit our data at fixed $C_{\mathrm{BSA}}$ for each polymer to the form

$$
\ln \left(D / D_{0}\right)=-a C_{\mathrm{PEO}}^{\vee}
$$

using a nonlinear least-squares grid-searching procedure. Table I gives values for $a$ and $v$ obtained from this approach. The constant $a$ is roughly proportional to the polymer molecular weight, going from $0.075-0.086$ to $0.22-0.27$ when the molecular weight of the polymer 
TABLE I

Parameters for Fits of the Diffusion Coefficient Data to the Power-Law Form $D / D_{0}=\exp \left(-a C^{\nu}\right)$

\begin{tabular}{cccccc}
\hline \multirow{2}{*}{$\begin{array}{c}\text { Protein } \\
\text { Concentration } \\
(\mathrm{g} / \mathrm{L})\end{array}$} & \multicolumn{2}{c}{$\begin{array}{c}\text { Polymer } \\
1 \times 10^{5}\end{array}$} & & \multicolumn{2}{c}{$\begin{array}{c}\text { Polymer } \\
3 \times 10^{5}\end{array}$} \\
\cline { 2 - 3 } \cline { 5 - 6 } & $a$ & $v$ & & $\alpha$ & $\nu$ \\
\hline 10 & 0.084 & 0.21 & & - & - \\
33 & 0.075 & 0.68 & & 0.24 & 0.82 \\
50 & 0.086 & 0.85 & & 0.24 & 0.77 \\
67 & - & - & & 0.27 & 0.68 \\
\hline
\end{tabular}

is tripled. In the $1 \times 10^{5}$ amu polymer solutions, $v$ seems to increase with increasing $C_{\mathrm{BSA}}$; an extrapolation of $v$ to $C_{\mathrm{BSA}} \rightarrow 0$ yields $v \approx 0$. For $C_{\mathrm{BSA}} \geq 33 \mathrm{~g} / \mathrm{L}, v$ is 0.68 or 0.85 ; these values are consistent with some of the published predictions for this parameter, such as the $v=$ $3 / 4$ predicted by deGennes. ${ }^{27}$ In the $3 \times 10^{5}$ amu polymer solutions, it is not clear that differences in $v$ are due to more than random scatter in the data; for the three values of $C_{\mathrm{BSA}}$ studied, $v$ has the average value 0.76 . This average is similar to the $v \approx 2 / 3$ reported by Langevin and Rondelez for BSA at nonzero concentration in the same water:polyethylene oxide mixtures. Langevin and Rondelez found $v$ to be concentration dependent and reported $v \approx 0.52$ as $C_{\mathrm{BSA}} \rightarrow 0$. In terms of the scaling models, $v$ indicates how a change in the concentration alters the typical distance between entanglement points; there is presently no clear theoretical interpretation of the amplitude $a$.

\section{CONCLUSIONS}

We have previously emphasized ${ }^{28}$ that the diffusion coefficient measured by light-scattering spectroscopy cannot be used to calculate the size of the diffusing particles, unless the solution is known to be dilute. The results given here show that $D$ and the Stokes-Einstein Eq. (1) also cannot be used to infer $R$ if the solvent is not well behaved. While water and highly viscous $(\sim 1000 \mathrm{cP})$ water:small molecular mixtures are in this sense well-behaved, ${ }^{29}$ polymer solutions are not. We find that the diffusion rate of a biopolymeric probe species through water:polyethylene oxide is appreciably faster than would be expected for a body of the known size of the probe and the macroscopic shear viscosity.

As has been shown elsewhere,$^{18}$ in some cases it is possible to establish useful calibration curves relating $D$ and $R$ for a given polymer solution. However, the interpretation of $D$ in terms of $R$ for a solute in a previously unstudied polymer solution appears at present to be fraught with hazard.

Comparison has been made with theoretical predictions that $D / D_{0}$ satisfies a scaling form such as Eq. (7); the free parameter $v$ was usually 
in the predicted range $1 / 2-1$. We did not determine the $R$ dependence of $a$, though Eqs. (4) and (6) predict $a \sim R .{ }^{1}$ Experimental results on polystyrene spheres in polymer solutions indicate that the $R$ dependence of $a$ is actually quite small, ${ }^{16-18}$ so the agreement between measured and predicted values of $v$ may be fortuitous. To pursue this question with more precision requires going to higher polymer concentrations, which is not possible in the system treated in this paper.

The support of National Institutes of Health Grant GM3182101 is gratefully acknowledged.

\section{References}

1. Jones, C. R., Johnson, C. S. Jr. \& Penniston, J. T. (1978) Biopolymers 17, 15811593.

2. Hall, R. S., Oh, Y. S. \& Johnson, C. S. Jr. (1980) J. Phys. Chem. 89, 756-767.

3. LaGatutta, K. J., Sharma, V. S., Nicoli, D. F. \& Kothari, B. K. (1981) Biophys. J. 33, 63-80.

4. Minton, A. P. \& Ross, P. D. (1978) J. Phys. Chem. 82, 1934-1938.

5. Gros, G. (1978) Biophys. J. 22, 453-468.

6. Keller, K. H., Canales, E. R. \& Yum, S. I. (1971) J. Phys. Chem. 75, 379-387.

7. Phillies, G. D. J., Benedek, G. B. \& Mazer, N. A. (1976) J. Chem. Phys. 65, 18831892.

8. Probstein, R. F., Leung, W. F. \& Alliance, Y. (1979) J. Phys. Chem. 83, 1228-1232.

9. Langevin, D. \& Rondelez, F. (1978) Polymer 19, 75-882.

10. Brown, W. \& Stilbs, P. (1983) Polymer 24, 188-192.

11. Turner, D. N. \& Hallett, R. F. (1976) Biochim Biophys. Acta 457, 305-312.

12. Laurent, T. C. \& Persson, H. (1964) Biochim Biophys. Acta 83, 141-147.

13. Laurent, T. C., Björk, I., Pietruszkiewicz, A. \& Persson, H. (1963) Biochim. Biophys. Acta 78, 351-359.

14. Laurent, T. C. \& Pietruszkiewicz, A. (1961) Biochim. Biophys. Acta 49, 258-264.

15. Lin, T.-H. \& Phillies, G. D. J. (1982) J. Phys. Chem. 86, 4073-4077.

16. Lin, T.H. \& Phillies, G. D. J. (1984) Macromolecules 17, 2050-2055.

17. Lin, T.-H. \& Phillies, G. D. J. (1984) J. Colloid Interface Sci. 100, 82-95,

18. Ullmann, G. \& Phillies, G. D. J. (1983) Macromolecules 16, 1947-1949.

19. Ogston, A. G., Preston, B. N. \& Wells, J. D. (1973) Proc. R. Soc. London Ser. A 333, 297-316.

20. Cukier, R. I. (1984) Macromolecules 17, 252-255.

21. Debye, P. \& Beuche, A. (1948) J. Chem. Phys. 16, 573-579.

22. Brinkman, H. C. (1947) Appl. Sci. Res. Sect. A 1, 27-35.

23. Freed, K. F. \& Edwards, S. F. (1974) J. Chem. Phys. 61, 3626-3633.

24. Freed, K. F. \& Edwards, S. F. (1975) J. Chem. Soc. Faraday Trans. 2 71, 20252029.

25. deGennes, P.-G. (1976) Macromolecules 9, 594-598,

26. Altenberger, A. R. \& Tirrell, M. (1984) J. Chem. Phys. 80, 2208-2213.

27. deGennes, P. G. (1976) Macromolecules 9, 587-593.

28. Phillies, G. D. J. (1974) J. Chem. Phys. 64, 976-983.

29. Phillies, G. D. J. (1981) J. Phys. Chem. 85, 2838-2843.

Received April 10, 1984

Accepted July 16, 1984 